\title{
Effect of stretching duration on active and passive range of motion in the lower extremity
}

\author{
Jennifer M Roberts, Karen Wilson
}

\begin{abstract}
Objectives-To investigate the effect of different durations of stretching (five or $\mathbf{1 5}$ seconds) on active and passive range of motion (ROM) in the lower extremity during a five week flexibility training programme.

Method-Twenty four university sport club members (19 men, five women), with a mean (SD) age of 20.5 (1.35) years, were randomly assigned to one of three groups (two treatment and one control). The two treatment groups participated in a static active stretching programme three times a week for a five week period, holding each stretch for a duration of either five or 15 seconds. The total amount of time spent in a stretched position was controlled. The five second group performed each stretch nine times and the 15 second group three times resulting in a total stretching time of 45 seconds for both groups for each exercise. The control group did not stretch. Active and passive ROM were determined during left hip flexion, left knee flexion, and left knee extension before and after the training programme using an inclinometer.
\end{abstract}

Results-Two factor within subject analysis of variance indicated no significant difference in ROM before and after the training programme for the control group. However, significant improvements in active and passive ROM $(p<0.05)$ were shown in both treatment groups after the five week training programme. Two factor analysis of variance with repeated measures and post hoc analysis showed significant differences between the treatment groups and the control group for the improvements observed in active $(p<0.05)$ and passive $(p<0.05)$ ROM. The five and 15 second treatment groups did not differ from one another when ROM was assessed passively, but significant differences were apparent for active ROM, with the 15 second group showing significantly greater improvements $(p<0.05)$ than the five second group.

Conclusion-These findings suggest that holding stretches for 15 seconds, as opposed to five seconds, may result in greater improvements in active ROM. However, sustaining a stretch may not significantly affect the improvements gained in passive ROM.

(Br f Sports Med 1999;33:259-263)

Keywords: range of motion; active; passive; flexibility; stretching duration; lower extremity
Flexibility can be defined as "the range of motion available at a joint or group of joints". ${ }^{1}$ This range of motion (ROM) can be achieved by either active muscular contraction, sometimes referred to as dynamic flexibility, ${ }^{2}$ or by the passive movement of the joint caused by some external force-for example, a coach or gravity (static flexibility).

Three methods of stretching to develop flexibility have emerged: ballistic stretching, static stretching, and proprioceptive neuromuscular facilitation techniques. All three methods have been shown to increase ROM immediately after stretching. ${ }^{3}$ However, because of the rapid and forceful nature of ballistic stretching and the theoretical potential to exceed the extensibility limits of a muscle, this method of increasing ROM has not been widely supported in the literature. ${ }^{4-6}$ Research investigating the biophysical properties of connective tissue and neurophysical factors affecting ROM has also brought into question the ability of ballistic stretching to promote long term improvements in flexibility. ${ }^{4} 6$

Several authors have identified proprioceptive neuromuscular facilitation techniques as being superior to other stretching methods for improving flexibility. ${ }^{38}$ However, as Bandy and Irion ${ }^{5}$ highlight, an experienced practitioner may be required to administer the techniques safely, reducing their suitability for most coaches and sports performers.

Consequently, static stretching has become the most widely used method for increasing ROM because of the simplicity of execution and lower potential for tissue trauma. ${ }^{3-5}$ While the literature is unanimous in its support for static stretching resulting in increased ROM, no consistency is apparent with regard to how long stretches should be held to obtain optimum benefits.

Recommendations for duration of stretching in flexibility training programmes range from five to 60 seconds, ${ }^{10} 11$ yet justifications for these selections have largely been absent. ${ }^{9}$ Madding $e t a l^{12}$ compared the effects of 15,45 , and 120 seconds of stretching on hip abduction, and reported that sustaining a stretch for 15 seconds was as effective as 120 seconds. These results, however, are based on one stretching session rather than an extended training programme.

Borms et $a l^{13}$ compared the effects of 10, 20, and 30 seconds of active static stretching on active coxo-femoral flexibility. The programme lasted for 10 weeks and consisted of two sessions a week. No significant differences were observed between the three groups, implying 
that a duration of 10 seconds static stretching is sufficient to elicit improvements in ROM.

Bandy and Irion ${ }^{9}$ compared the effects of stretching durations of 15, 30, and 60 seconds on hamstring flexibility. The training programme lasted six weeks and involved subjects stretching passively five times a week. Measures of passive knee extension before and after the training programme showed significantly greater improvements in ROM in the 30 and 60 second groups than the 15 second group, but no differences existed between the improvements shown by the 30 and 60 second groups.

It is clear that there are limited and conflicting findings in this area. Comparison and subsequent conclusions are difficult because of the lack of consistency in the selection of stretching methods employed and whether active or passive movement is assessed. In the literature reviewed, no study has indicated attempts to control for total amount of time spent stretching while manipulating stretch duration. This factor may influence the improvements observed in ROM after a flexibility training programme continued over a number of weeks. Holding stretches for 60 seconds over a six week period would result in a much longer total time spent stretching than holding stretches for only 10 seconds. This larger total amount of time spent in a stretched position may elicit greater improvements in ROM irrespective of the duration of the individual stretches. Also differences in active and passive ROM have not been considered. Therefore the purpose of this study is to compare the effect of stretching duration on both active and passive ROM while controlling the total amount of time spent in a stretched position.

\begin{abstract}
Method
SUBJECTS

Twenty four university sports team/club members, with a mean (SD) age of 20.5 (1.35) years, volunteered to act as subjects for the study (19 men and five women). Subjects were randomly assigned to either a control group or one of two treatment groups with eight subjects in each group. The treatment groups were required to complete a static active stretching programme, with one group holding each stretch for a duration of 15 seconds (seven men and one woman) and the other group holding each stretch for a five second duration (six men and two women). The control group did not stretch (six men and two women). All subjects agreed to maintain their normal exercise and activity levels for the duration of the study.
\end{abstract}

Table 1 Summary of stretching protocols

\begin{tabular}{llllll}
\hline Treatment & $\begin{array}{l}\text { Duration of } \\
\text { stretch } \\
\text { (seconds) }\end{array}$ & $\begin{array}{l}\text { Rest interval } \\
\text { (seconds) }\end{array}$ & $\begin{array}{l}\text { Number of } \\
\text { repetitions of } \\
\text { stretch }\end{array}$ & Number of sets & $\begin{array}{l}\text { Total time } \\
\text { spent in a } \\
\text { strextched } \\
\text { position } \\
\text { (seconds) }\end{array}$ \\
\hline 1 & 15 & 15 & 3 & 1 & 45 \\
2 & 5 & 15 & 3 & 3 & 45 \\
\hline
\end{tabular}

PROCEDURES CARRIED OUT BEFORE TESTING Before each subject was tested, the time, date, and temperature of the room was recorded. A standardised 10 minute aerobic warm up (intensity $60-80 \%$ heart rate maximum) involving jogging and arm and hip circling was then performed.

ASSESSMENT OF EACH SUBJECT

ROM was assessed using both active and passive movements to determine dynamic and static flexibility of the left lower limb, specifically, during flexion at the hip joint and flexion and extension at the knee joint. Assessment of ROM at each joint location followed procedural guidelines recommended by MacDougall et $a l^{14}$ for the determination of start and end positions. The end of passive ROM was determined once the tester felt resistance or the subject vocalised discomfort. Angular displacement was measured using an inclinometer ${ }^{15}$ (MIE-Medical Research Ltd, Leeds, UK). All movements took place in the sagittal plane after the assumption of the fundamental anatomical position. Anatomical landmarks were identified and the goniometer then positioned. Markings were made and the distance of the goniometer from the joint centre was recorded for future reference. The goniometer was removed and the movement was then performed. The goniometer was then replaced in the same "marked" position and the measurement recorded. Each movement was repeated three times and recorded in degrees. The same tester performed all measurements and was blind to which group the subject belonged.

\section{TRAINING PROGRAMME}

After the pre-test, each subject was issued with a training programme that involved stretching three times a week for a five week period. The programme contained details of six static active stretches of the lower extremity, two stretches for the hamstrings and hip extensors, two stretches for quadriceps and hip flexors, and two for the lower leg. Each stretch was held for five or 15 seconds depending on the group to which the subject had been randomly assigned. To control for the total time spent in a stretched position, repetitions, rest intervals, and the number of sets for each stretch performed were different for each treatment group. This information was outlined for each subject in the programme and is highlighted in table 1 .

The programme also contained safety guidelines and details of the warm up procedure. The warm up was identical with that performed before the initial assessment of ROM. Briefing sessions were co-ordinated in which visual demonstrations and individual assistance were provided to ensure all subjects were confident in the requirements of the programme and competent in the execution of the stretches. No set time of day was specified at which the stretch had to be performed, but there was at least one day of rest between sessions. 
PROCEDURES FOR REASSESSMENT OF ROM

After the five week period, ROM was reassessed with measurement procedures replicating those used during the initial assessment. All three groups of subjects were tested under the same environmental conditions on the same day and time as their respective pre-test.

STATISTICAL ANALYSIS

The mean (SD) of the three measurements taken for each movement was used in the analysis of data. Means (SEM) were calculated for each of the groups, for each movement before and after the training programme under active and passive assessment.

Two factor within subject analysis of variance with follow up Bonferroni adjusted $t$ tests were selected to identify any significant differences between the pre and post test scores within each group. Change in active and passive ROM was calculated for each subject using pre- and post-test scores for each movement performed (change $=$ post-test score pre-test score). These data were then used in two factor analysis of variance with repeated measures on one factor with post hoc Tukey's Honestly Significant Difference (HSD) tests to identify any significant differences in change in active and passive ROM between each of the three groups. $p \leqslant 0.05$ was accepted as the significance level for analysis.

\section{Results}

Mean (SEM) scores for active (table 2) and passive (table 3) ROM before and after the training programme at each joint site were calculated for the control and the two treatment groups.

The change in ROM was calculated for each joint movement using the pre- and post-test scores for each subject. These values represent the deviation in degrees between ROM achieved before and after the training programme and are summarised as mean change in ROM for each group in table 4 .

Two factor within subject analysis of variance showed no significant difference in ROM before and after the training programme for the control group, indicating that no significant improvement in active or passive ROM had occurred for this group during the duration of the study. Differences were observed for the 15 second $(p<0.05)$ and five second $(p<0.05)$ treatment groups, indicating that the five week flexibility training programme had a significant effect on both active and passive ROM.

Two factor analysis of variance with repeated measures showed significant differences between the three groups, indicating that the duration of stretch employed during the training programmes significantly affected the improvements observed in both active and passive ROM.

Post hoc Tukey's HSD tests on the results showed that the group holding stretches for 15 seconds and the group holding stretches for five seconds significantly improved ROM when compared with the control group. This was the case for both active $(\mathrm{p}<0.05)$ and passive $(\mathrm{p}<0.05)$ assessment of ROM. There was no significant differences observed between the improvements made by either the 15 second or the five second group in passive ROM. However, significant differences were shown between the two treatment groups when assessed actively. The group holding the stretches for 15 seconds showed significantly greater improvements $(\mathrm{p}<0.05)$ in active ROM than the group holding the stretches for only five seconds. This was true for all joint movements assessed. Figure 1 provides a graphical representation of these findings using the results for left hip flexion as an illustration of the trend observed.

Table 2 Mean (SEM) active range of movement for each variable (in degrees) before and after the training programme for all groups

\begin{tabular}{|c|c|c|c|c|c|c|}
\hline \multirow[b]{2}{*}{ Variable } & \multicolumn{2}{|l|}{ Control } & \multicolumn{2}{|l|}{15 seconds } & \multicolumn{2}{|l|}{ Five seconds } \\
\hline & Before & After & Before & After & Before & After \\
\hline Hip flexion & $93.71(6.65)$ & $94.08(6.69)$ & $94.50(5.01)$ & $103.00(5.05)$ & $92.29(4.72)$ & $97.17(4.54)$ \\
\hline Knee flexion & $135.33(5.44)$ & $135.67(5.48)$ & $134.79(4.28)$ & $142.33(4.10)$ & $132.08(4.91)$ & $136.87(4.74)$ \\
\hline Knee extension & $92.16(3.65)$ & $92.79(3.39)$ & $91.75(2.36)$ & $99.50(2.24)$ & $88.75(2.50)$ & $93.33(2.52)$ \\
\hline
\end{tabular}

Table 3 Mean (SEM) passive range of movement for each variable (in degrees) before and after the training programme for all groups

\begin{tabular}{|c|c|c|c|c|c|c|}
\hline \multirow[b]{2}{*}{ Variable } & \multicolumn{2}{|l|}{ Control } & \multicolumn{2}{|l|}{15 seconds } & \multicolumn{2}{|l|}{ Five seconds } \\
\hline & Before & After & Before & After & Before & After \\
\hline Hip flexion & $108.37(6.20)$ & $109.04(5.97)$ & $107.62(4.68)$ & $113.79(4.61)$ & $105.71(4.09)$ & $111.79(3.27)$ \\
\hline Knee flexion & $145.04(5.18)$ & $145.62(5.09)$ & $146.96(4.15)$ & $153.46(3.91)$ & $143.58(4.47)$ & $149.00(3.74)$ \\
\hline Knee extension & $102.21(3.24)$ & $102.50(3.41)$ & $101.79(3.10)$ & $107.58(2.97)$ & $100.87(2.83)$ & $106.21(2.14)$ \\
\hline
\end{tabular}

Table 4 Summary of the mean (SEM) change in range of movement for each of the groups (in degrees) under both active and passive assessment

\begin{tabular}{|c|c|c|c|c|c|c|}
\hline \multirow[b]{2}{*}{ Variable } & \multicolumn{2}{|l|}{ Control } & \multicolumn{2}{|l|}{15 seconds } & \multicolumn{2}{|l|}{ Five seconds } \\
\hline & Active & Passive & Active & Passive & Active & Passive \\
\hline Hip flexion & $0.37(0.50)$ & $0.67(0.48)$ & $8.50(0.38)$ & $6.16(0.69)$ & $4.87(0.82)$ & $6.00(1.46)$ \\
\hline Knee flexion & $0.33(0.39)$ & $0.58(0.33)$ & $7.54(0.59)$ & $6.50(0.48)$ & $4.79(0.57)$ & $5.41(0.83)$ \\
\hline Knee extension & $0.63(0.44)$ & $0.29(0.39)$ & $7.75(0.72)$ & $5.79(0.58)$ & $4.58(0.39)$ & $5.33(1.04)$ \\
\hline
\end{tabular}




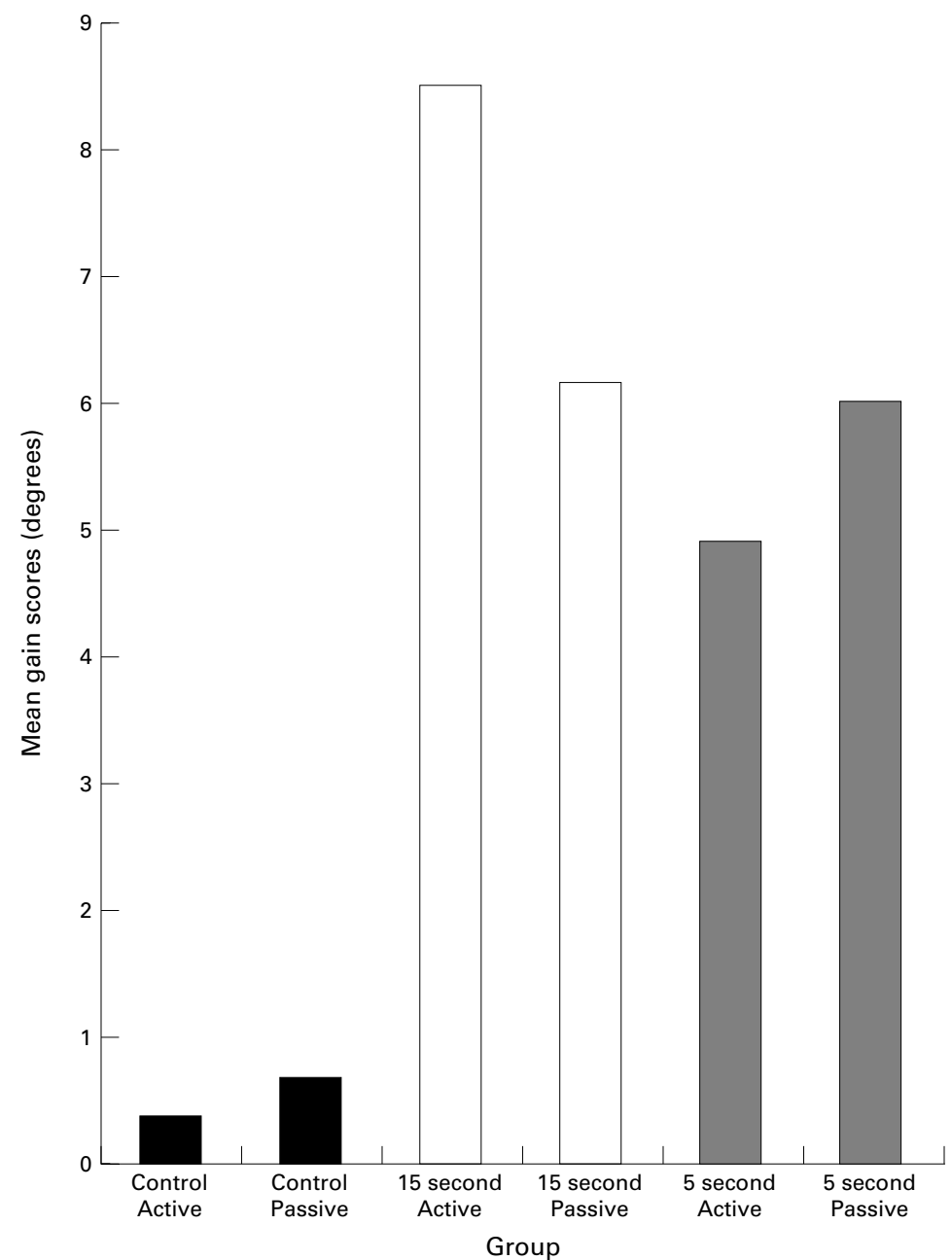

Figure 1 Mean gain scores for active and passive range of motion during hip flexion for all groups.
Differences were apparent between the five and 15 second groups when ROM was assessed actively. These findings indicate that holding an active stretch for 15 seconds results in greater improvements in active ROM compared with holding the stretch for only five seconds. Borms et $a l^{13}$ followed a similar protocol to this study, using an active stretching programme and assessing active ROM. However, results indicated no significant difference between stretches of 10,20 , and 30 seconds. The researchers speculated that 10 seconds of static stretching was sufficient to elicit a Golgi tendon organ response and therefore provide an effective flexibility training stimulus. The differences observed in active ROM between the five and 15 second groups in the present study could be potentially explained by stimulation of muscle proprioceptors. A stretching duration of five seconds resulted in significantly smaller improvements in active ROM than a duration of 15 seconds, suggesting that five seconds may not be long enough to elicit a Golgi tendon organ response. However, the lack of any differences in passive ROM between the five and 15 second groups suggests that this is not the case.

A possible explanation for the findings of this study is a potential connection with the development of increased strength as a result of an active stretching programme. While actively stretching, subjects would be experiencing an exercise overload in the agonist muscle group that is required to contract to sustain the stretch position. The 15 second group, when compared with the five second group, would experience an increased exercise overload, which would result in greater improvements in muscular strength for the subjects holding stretches for the longer duration. The increase in muscular strength would be manifested during active assessment of ROM but not passive assessment.

The implications of these findings could be important in training programme design for athletes wishing to develop flexibility. The findings suggest that an athlete who participates in a sport that primarily demands a large functional ROM - that is, dynamic flexibilityshould employ active stretching techniques with stretches being held. The findings may also have implications for clinicians incorporating stretching activities into rehabilitation programmes.

Further research is needed to investigate the effect of static active stretching on both muscular strength and ROM, in order to determine optimal stretching duration. A static passive stretching programme has not been examined by this study. This area also needs investigation to provide a fuller understanding of the relation between training stimulus and optimal improvements in ROM. Future research needs to look at the effects of long term training programmes with stretching duration ranging from five to 120 seconds or longer at multiple joint sites. Stretching and assessment techniques need to encompass both active and passive movements, and the issue of total time time spent in a stretched position during the entire training programme. 
spent in a stretched position needs to be considered.

CONCLUSION

This study indicates that a five week active stretching programme significantly increases active and passive ROM in the lower extremity. Findings indicate that increasing the duration of stretch from five to 15 seconds does not significantly influence increase in passive ROM. Further research is necessary to investigate whether this trend is observed when longer stretching duration is used. Stretching for a duration of 15 seconds produces significantly greater improvements in active ROM than stretching for five seconds. However, speculations can only be made about possible explanations for this relation. Further research is necessary involving assessment of active and passive ROM and muscular strength in response to long term flexibility training programmes using a larger range of stretching duration in order to determine the optimum.

We would like to thank Helen Allinson who served as a research assistant in this study.

1 Corbin CB, Lindsey R. Concepts of physical fitness (with laboratories). Dubuque, IA: Brown \& Benchmark, 1994:77.

2 McNaught Davis P. Flexibility: how to understand it, how to achieve it. London: Partridge Press, 1991.
3 Sady SP, Wartman M, Blanke D. Flexibility training: ballisic static or proprioceptive neuromuscular facilitation? tic, static or proprioceptive neurom
Arch Phys Med Rehabil 1982;63:261-3.

4 Alter MJ. Science of stretching. Champaign, IL: Human Kinetic Books, 1988.

5 Bandy WD, Irion JM. The effect of time on static stretch on the flexibility of the hamstring muscles. Phys Ther 1994;74: 845-50.

6 Anderson B, Burke ER. Scientific, medical and practical aspects of stretching. Clin Sports Med 1991;10:63-86.

7 Sapega AA, Quedenfeld TC, Moyer RA, et al. Biophysical factors in range of movement exercise. Physician and Sports Medicine 1981;9:57-65.

8 Shellock FG, Prentice WE. Warming-up and stretching for improved physical performance and prevention of sportsimproved physical performance and preventic

9 Entyre BR, Abraham LD. Gains in range of ankle dorsiflexion using three popular stretching techniques. Am F Phys Med 1986;65:189-96.

10 Liemohn W. Flexibility/range of motion. In: American College of Sports Medicine, eds. Resource manual for guidelines for exercise testing and prescription. London: Lea \& Febiger, 1993.

11 Moffatt RJ. Strength and flexibility considerations for exercise prescription. In: American College of Sports Medicine, eds. Resource manual for guidelines for exercise testing and prescription. London: Lea \& Febiger, 1993.

12 Madding SW, Wong JG, Hallum A, et al. Effects of duration or passive stretching on hip abduction range of motion. $\mathcal{f}$ or passive stretching on hip abduction
Orthop Sports Phys Ther 1987;8:409-16.

13 Borms J, Van Roy P, Santans J-P, et al. Optimal duration of static stretching exercises for improvement of coxo-femoral flexibility. F Sports Sci 1987;5:39-47.

14 MacDougall JD, Wenger HA, Green HJ. Physiological testing of the high-performance athlete. 2nd ed. Leeds: Human Kinetic Publishers, 1991.

15 Maud PJ, Cortez-Cooper MY. Static techniques for the evaluation of joint range of motion. In: Maud PJ, Foster C, eds. Physiological assessment of human fitness. Champaign, IL: Human Kinetics, 1995:221-42.

16 Cornelius WL, Hagemann RW, Jackson AW. A study on placement of stretching within a workout. $\mathcal{F}$ Sports Med and Phys Fitness 1988;28:234-6.

\section{Take home message}

Holding an active stretch for longer may not significantly influence increase in passive ROM. However, stretch duration may have a significant effect on improvements in active ROM. This may have implications for the design of flexibility training programmes and rehabilitation programmes incorporating stretching activities. 